\title{
The effect of decision weights in bargaining problems
}

Citation for published version (APA):

Peters, H. J. M., \& Koebberling, V. (2002). The effect of decision weights in bargaining problems.

METEOR, Maastricht University School of Business and Economics. METEOR Research Memorandum No. 064 https://doi.org/10.26481/umamet.2002064

Document status and date:

Published: 01/01/2002

DOI:

10.26481/umamet.2002064

Document Version:

Publisher's PDF, also known as Version of record

\section{Please check the document version of this publication:}

- A submitted manuscript is the version of the article upon submission and before peer-review. There can be important differences between the submitted version and the official published version of record.

People interested in the research are advised to contact the author for the final version of the publication, or visit the DOI to the publisher's website.

- The final author version and the galley proof are versions of the publication after peer review.

- The final published version features the final layout of the paper including the volume, issue and page numbers.

Link to publication

\footnotetext{
General rights rights.

- You may freely distribute the URL identifying the publication in the public portal. please follow below link for the End User Agreement:

www.umlib.nl/taverne-license

Take down policy

If you believe that this document breaches copyright please contact us at:

repository@maastrichtuniversity.nl

providing details and we will investigate your claim.
}

Copyright and moral rights for the publications made accessible in the public portal are retained by the authors and/or other copyright owners and it is a condition of accessing publications that users recognise and abide by the legal requirements associated with these

- Users may download and print one copy of any publication from the public portal for the purpose of private study or research.

- You may not further distribute the material or use it for any profit-making activity or commercial gain

If the publication is distributed under the terms of Article $25 \mathrm{fa}$ of the Dutch Copyright Act, indicated by the "Taverne" license above, 


\title{
The Effect of Decision Weights in Bargaining Problems*
}

\author{
Veronika Köbberling ${ }^{1} \quad$ Hans Peters ${ }^{2}$
}

March 2002, final version

${ }^{1}$ Department of Quantitative Economics, University of Maastricht, P.O. Box 616, 6200 MD Maastricht. Telephone $+31-43-3883834$, telefax $+31-43-3884874$.

E-mail address: v.koebberling@ke.unimaas.nl.

${ }^{2}$ Corresponding author. Department of Quantitative Economics, University of Maastricht, P.O. Box 616, 6200 MD Maastricht. Telephone +31-43-3883834, telefax +31-43-3884874.

E-mail address: h.peters@ke.unimaas.nl.

Running title: Decision weights in bargaining

*Veronika Köbberling thanks the Dutch Science Foundation NWO for financial support under grant 425-11-003. The authors thank Peter Wakker, the associate editor and a referee for many helpful comments and suggestions. The usual disclaimer applies. 


\begin{abstract}
Bargaining problems are considered where the preferences of the bargainers deviate from expected utility but can be modelled according to rank dependent utility theory. Under rank dependent utility both the utility function and the probability weighting function influence the risk attitude of a decision maker. The same definition of risk aversion leads to two forms of risk aversion: utility risk aversion and probabilistic risk aversion. The main finding is that these two forms can have surprisingly opposite consequences for bargaining solutions that exhibit a weak monotonicity property. In particular, in a large class of bargaining problems both increased utility risk aversion and decreased probabilistic risk aversion of the opponent are advantagous for a player. This is demonstrated for the Kalai-Smorodinsky bargaining solution. The Nash bargaining solution does not behave regularly in this respect. Keywords: Bargaining, risk aversion, rank dependent utility. Journal of Economic Literature Classification numbers: 026, 022.
\end{abstract}




\section{Introduction}

A bargaining problem is described by a set of potential outcomes, including a so-called disagreement outcome. The bargainers try to reach agreement on one of the outcomes or on a lottery between these outcomes. If they fail, the disagreement outcome results. A bargaining solution suggests an agreement outcome or lottery for each possible bargaining problem. In this paper, the socalled welfarist approach to bargaining is adopted. In this approach, the solution to the bargaining problem depends exclusively on its image in utility space.

The axiomatic approach to two-person bargaining problems was initiated by Nash [9], under the assumption that the two bargainers maximize expected utility. In expected utility, risk aversion is an important empirical phenomenon. Yaari [21] provides an intuitive definition of risk aversion and shows that under expected utility one decision maker is more risk averse than another if and only if the utility function of the former is a strictly increasing concave transformation of that of the latter. An interesting question, first studied by Kannai [7] and Kihlstrom, Roth, and Schmeidler [8], concerns the effect of risk aversion on the outcomes predicted by bargaining solutions. Their answers to this question confirm a plausible intuition: it is advantagous to play against a more risk averse bargainer. These results are driven by the fact that a more risk averse bargainer has a more concave utility function. Therefore, utility at low levels increases at a faster rate and satisfaction may appear faster. Because most bargaining solutions are sensitive to this, a more risk averse bargainer may be easier to satisfy, which is advantagous for the opponent (cf. Peters, [10]).

Expected utility as a normative basis for decision making is appealing, but as empirical findings show, it is descriptively unsatisfactory. Therefore, alternative models have been developed of which rank dependent utility (Quiggin, [11]), which extends expected utility by allowing for distortion of probabilities, is the best known.

Our paper considers bargaining problems where the bargainers' preferences can be represented by rank dependent utility. In this model Yaari's concept of increased risk aversion has still the same natural meaning: a more risk averse person prefers less lotteries over each riskless alternative. In contrast with expected utility, however, not only the utility function but also a probability weighting function determines the risk attitude of a decision maker. An elegant and simple characterization of increased risk aversion as in the case of expected utility is not yet available. Chateauneuf and Cohen [2] provide some partial results. Chew, Karni, and Safra [3] characterize aversion to mean-preserving spreads, which is more restrictive than risk aversion. 
In the present paper, two factors of risk aversion are distinguished: utility risk aversion (as in the expected utility model), associated with the utility function; and probabilistic risk aversion, associated with the probability weighting function. In our application to bargaining we concentrate on the latter, because the results for the former are closely related to those in expected utility. As in the related literature, the central question is: is it advantagous or disadvantagous to bargain with a more risk averse person? In Section 3, we investigate the impact of both utility and probabilistic risk aversion for the Kalai-Smorodinsky bargaining solution (Kalai and Smorodinsky, [5]). The main finding is that in a large class of bargaining problems, it is advantagous to have a less probabilistically risk averse, or a more utility risk averse opponent. The known effect of risk aversion for the expected utility case (cf. Kihlstrom et al., [8]) is a special case of the latter result. Since both forms of risk aversion arise from the same concept of comparative risk aversion, this contrast is rather surprising. On closer inspection, however, it is not counterintuitive that increased probabilistic risk aversion of the opponent could be bad for a player, because such an opponent might insist on larger probabilities for good alternatives and thereby reduce the other player's utility.

The two results also have some strategic implications, if it is assumed that bargainers can be dishonest about their true preferences and pretend to evaluate lotteries by means of a different utility or probability weighting function. Specifically, it does not pay to pretend to be less probabilistically risk averse, or more utility risk averse, but it might sometimes pay to pretend to be more probabilistically or less utility risk averse. For the expected utility case related papers in this direction are Crawford and Varian [4] and Sobel [17,18].

Although our focus is on the Kalai-Smorodinsky solution the main results in this paper can be extended to solutions that exhibit some form of monotonicity. Typically, these do not include the Nash bargaining solution. If this solution is used, a more probabilistically risk averse opponent might be better but also worse for a player. We justify the use of the Kalai-Smorodinsky solution in bargaining under rank dependent utility by showing that the axiomatization of Kalai and Smorodinsky [5] still makes sense (see Section 4).

Axiomatic bargaining without expected utility has been studied in other articles (Rubinstein, Safra, and Thomson, [13]; Safra and Zilcha, [14]; Safra, Zhou, and Zilcha, [15]; Burgos, Grant, and Kaji, [1]; and Volij and Winter, [22]). The paper of Volij and Winter [22] is closest to the present paper. It applies Yaari's dual theory of choice to bargaining problems where the set of alternatives consists of all distributions of one unit of a perfectly divisible good, and uses the stronger notion of aversion to mean-preserving spreads to study changes in the Nash solution. Shalev [16] has studied 
the effect of loss aversion-see also Section 5 .

The proofs are collected in the Appendix.

\section{Preliminaries}

\subsection{Rank dependent utility}

Throughout, the set $A$ of riskless alternatives is a nonempty compact topological space. A lottery is a probability measure with finite support on $A$, typically denoted by $P=\left(p^{1}, a^{1} ; \ldots ; p^{n}, a^{n}\right)$ where $a^{1}, \ldots, a^{n} \in A, p^{i} \geq 0$ for all $i$, and $\sum_{i=1}^{n} p^{i}=1$. The set of all lotteries is $L(A)$. A riskless alternative $a \in A$ is identified with the lottery $(1, a) \in L(A)$.

A utility function $U: A \rightarrow \mathbb{R}$ is a continuous function that assigns to each riskless alternative a real number, expressing its value for a decision maker. A weighting function is a continuous, strictly increasing function $w:[0,1] \rightarrow[0,1]$ with $w(0)=0$ and $w(1)=1$, representing the decision maker's personal appraisal of probabilities. For a decision maker with utility function $U$, the preferences over $L(A)$ can be modelled by rank dependent utility (RDU) if there exists a weighting function $w$, such that preferences are represented by the functional $R D U: L(A) \rightarrow \mathbb{R}$ defined by

$$
\begin{aligned}
R D U(P)= & w\left(p^{\rho(1)}\right) U\left(a^{\rho(1)}\right)+ \\
& \sum_{i=2}^{n}\left[w\left(p^{\rho(1)}+\cdots+p^{\rho(i)}\right)-w\left(p^{\rho(1)}+\cdots+p^{\rho(i-1)}\right)\right] U\left(a^{\rho(i)}\right),
\end{aligned}
$$

where $\rho$ is a permutation that orders the riskless alternatives in the lottery from best to worse, i.e., $U\left(a^{\rho(1)}\right) \geq \ldots \geq U\left(a^{\rho(n)}\right)$ (cf. Quiggin, [11]). Expected utility holds if $w(p)=p$ for all $p \in[0,1]$.

Rank dependent utility, in contrast to expected utility, permits transformations of probabilities. An alternative is weighted by the transformed probability of obtaining the alternative or something better minus the transformed probability of obtaining something better.

\subsection{Comparative risk aversion}

Yaari [20] introduced a natural definition of risk aversion. A decision maker is more risk averse than another one, if whenever the former (weakly) prefers a lottery to a riskless alternative the latter also does. In expected utility it is a well known result that one decision maker is more risk averse than a second one if the utility function of the first is a strictly increasing concave transformation of that of the second. Under rank dependent utility, a second component influences the risk attitude, 
namely, the weighting function. In the lemma below and in the rest of the paper we consider these two components separately, by keeping one fixed and varying the other.

Definition 2.1 Let the preferences over $L(A)$ of two decision makers ( $D M$ and $\widetilde{D M}$ ) be represented by RDU-functionals $R D U$ and $\widetilde{R D U} \cdot \widetilde{D M}$ is more risk averse than $D M$ if for all $a \in A$ and all $P \in L(A)$ with $\widetilde{R D U}(P) \geq \widetilde{R D U}(a)$ also $R D U(P) \geq R D U(a)$.

Lemma 2.2 Let the preferences over $L(A)$ of $D M$ and $\widetilde{D M}$ be represented by $R D U$ and $\widetilde{R D U}$ with utility functions $U$ and $\widetilde{U}$ and weighting functions $w$ and $\widetilde{w}$, respectively.

1. If $w=\widetilde{w}$ then $\widetilde{D M}$ is more risk averse than $D M$ if and only if there exists a concave strictly increasing function $k: \mathbb{R} \rightarrow \mathbb{R}$ such that $\widetilde{U}=k \circ U$.

2. If $U=\alpha \widetilde{U}+\beta$ for $\alpha, \beta \in \mathbb{R}, \alpha>0$ then $\widetilde{D M}$ is more risk averse than $D M$ if $\widetilde{w}(p) \leq w(p)$ for all $p \in[0,1]$. In particular, if $U=\widetilde{U}$ this implies that $\widetilde{R D U}(P) \leq R D U(P)$ for all $P \in L(A)$.

If $A$ is connected, the "only if" implication in part 2 of Lemma 2.2 also holds. In the situation of Lemma 2.2, part 1, we say that decision maker $\widetilde{D M}$ is more utility risk averse than decision maker $D M$; whereas in the situation of Lemma 2.2, part 2, we say that $\widetilde{D M}$ is more probabilistically risk averse than $D M$. Although the above result is well known and simple to prove, it was not yet stated as in Lemma 2.2. Part 1 of the lemma is a generalization of Yaari's result [20] for expected utility; a predecessor of part 2 can be found in Yaari [21]. A proof can also be deduced from Chateauneuf and Cohen [2]. A general characterization of comparative risk aversion that allows for varying utility and weighting functions at the same time, is not yet available.

\subsection{Bargaining problems and solutions}

As before, a nonempty compact topological space $A$ is the set of riskless alternatives. Let $R D U_{1}$ and $R D U_{2}: L(A) \rightarrow \mathbb{R}$ be the RDU-functionals of two bargainers with utility functions $U_{1}$ and $U_{2}$ and weighting functions $w_{1}$ and $w_{2}$, respectively. A designated element $\bar{a} \in A$ is the disagreement alternative. We assume throughout that there is a lottery $P \in L(A)$ with $R D U_{1}(P)>U_{1}(\bar{a})$ and $R D U_{2}(P)>U_{2}(\bar{a})$. The two bargainers try to reach an agreement in $L(A)$. If they fail, $\bar{a}$ results. The quadruple $\left(A, \bar{a}, R D U_{1}, R D U_{2}\right)$ is called a (two-person) bargaining problem. $\mathbb{B}$ is the set of all bargaining problems. 
For a bargaining problem $B=\left(A, \bar{a}, R D U_{1}, R D U_{2}\right)$, define

$$
\begin{aligned}
& S(B)=\operatorname{cl}\left\{\left(R D U_{1}(P), R D U_{2}(P)\right): P \in L(A)\right\} \text { and } \\
& d(B)=\left(U_{1}(\bar{a}), U_{2}(\bar{a})\right)
\end{aligned}
$$

where 'cl' denotes the (topological) closure. The point $d(B)$ is called the disagreement outcome. The set $S(B)$ is called the feasible set of $B$. It is a compact subset of $\mathbb{R}^{2}$. In contrast to the case of bargaining problems under expected utility, examples (with infinite $A$ ) can be constructed where the set $\left\{\left(R D U_{1}(P), R D U_{2}(P)\right): P \in L(A)\right\}$ is not closed.

We define

$$
\mathbb{S}=\{(S, d): S=S(B) \text { and } d=d(B) \text { for some } B \in \mathbb{B}\} .
$$

A bargaining solution is a map $F: \mathbb{S} \rightarrow \mathbb{R}^{2}$ that assigns to each $(S, d) \in \mathbb{S}$ an element of $S$. In the rest of the paper, we also write $F(B)$ instead of $F(S(B), d(B))$.

\subsection{Feasible sets of bargaining problems}

Under expected utility, the set of all possible feasible sets is the set of all compact convex sets in $\mathbb{R}^{2}$. Because of continuity of the weighting functions, a feasible set $S(B)$ is connected under rank dependent utility, but it is not necessarily convex. It does, however, satisfy a weaker form of convexity, as formulated in the following lemma.

For any points $s$ and $t$ in $\mathbb{R}^{2}$ denote by $[s, t]$ the straight line segment with $s$ and $t$ as endpoints, i.e., the convex hull of $s$ and $t$. The vector inequality $t>s$ means $t_{i}>s_{i}$ for $i=1,2$, and $t \geq s$ means $t_{i} \geq s_{i}$ for $i=1,2$.

Lemma 2.3 Let $(S, d) \in \mathbb{S}$. Then for all $s, t \in S$ with $s_{1}=t_{1}$ or $s_{2}=t_{2}$ we have $[s, t] \subseteq S$.

This lemma implies in particular that a feasible set $S$ is not only connected but also simply connected: it contains no 'holes' (cf. Lemma A.1 in the Appendix).

For $(S, d) \in \mathbb{S}$ the Pareto optimal set $P(S)$ is defined by

$$
P(S)=\{s \in S: \text { there exists no } t \in S \text { with } t \geq s \text { and } t \neq s\},
$$

Instead of $P(S(B))$ we also write $P(B)$.

The following lemma implies that the Pareto optimal set is connected. 
Lemma 2.4 Let $(S, d) \in \mathbb{S}$. Then there is a closed interval $I \subset \mathbb{R}$ and a continuous strictly decreasing function $f: I \rightarrow \mathbb{R}$ such that $P(S)$ is the graph of $f$, that is,

$$
P(S)=\{(t, f(t)): t \in I\}
$$

Lemma 2.4 says that Pareto optimal subset of a feasible set $S$ behaves nicely: there are no indentations nor even horizontal or vertical line segments. Analogous results can be derived for the other parts of the boundary of $S$ (cf. Lemma A.3 in the Appendix). Lemma 2.3 implies that also the possibly flat parts in the north, east, south, and west cannot have indentations.

The next lemma provides a partial answer to the converse question: Which nonempty compact subsets $S$ of $\mathbb{R}^{2}$ can be generated as feasible sets of bargaining problems under rank dependent utility? The lemma says that every connected compact Pareto optimal set can be generated by an underlying bargaining problem.

Lemma 2.5 Let $I \subset \mathbb{R}$ be a nonempty closed interval and let $f: I \rightarrow \mathbb{R}$ be a continuous strictly decreasing function. Then there is a $B \in \mathbb{B}$ such that $P(B)$ is the graph of $f$, that is,

$$
P(B)=\{(t, f(t)): t \in I\}
$$

\subsection{The Kalai-Smorodinsky bargaining solution}

For $(S, d) \in \mathbb{S}$, the utopia point is defined by:

$$
u(S, d)=\left(\max \left\{s_{1}: s \in S, s \geq d\right\}, \max \left\{s_{2}: s \in S, s \geq d\right\}\right)
$$

For $B \in \mathbb{B}$ we also write $u(B)$ instead of $u(S(B), d(B)$ ). The Kalai-Smorodinsky solution $K S$ (Kalai and Smorodinsky, [5]) assigns to each $(S, d) \in \mathbb{S}$ the maximal point of $S$ on the line segment with $d$ and $u(S, d)$ as endpoints. It is well defined because $S$ is compact. By Lemma $2.4, K S(S, d)$ is a Pareto optimal point for every $(S, d) \in \mathbb{S}$.

This is an adaptation of the definition of $\mathrm{KS}$ for expected utility to rank dependent utility. Under expected utility, the Kalai-Smorodinsky solution can be justified and characterized by a set

of axioms (Kalai and Smorodinsky, [5]). This axiomatization can be extended to the rank dependent utility case, as we will show in Section 4 below. 


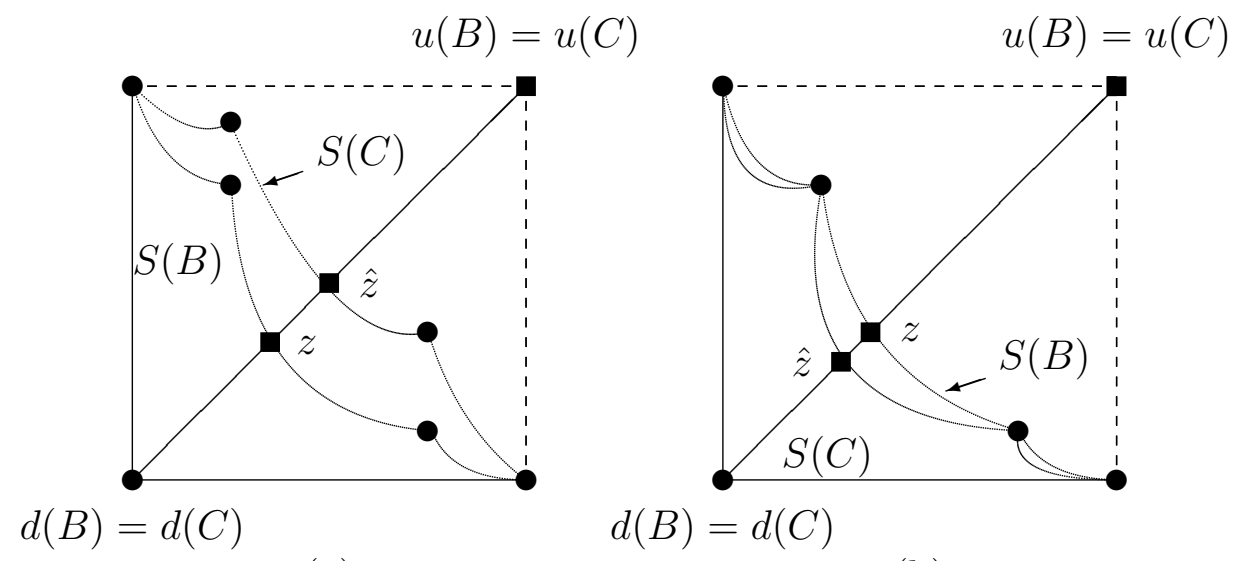

(a)

(b)

Figure 1: Diagram (a) illustrates Theorem 3.1 and diagram (b) illustrates Theorem 3.2. In both diagrams, $z=K S(B)$ and $\hat{z}=K S(C)$. The solid dots correspond to riskless alternatives.

\section{Sensitivity of the KS bargaining solution to risk}

We investigate the sensitivity of the Kalai-Smorodinsky bargaining solution to changes in the risk attitude of the bargainers. We first assume that both bargainers weakly prefer all riskless alternatives to the disagreement alternative, a condition which will be weakened in Section 3.4. We examine the changes in the outcome suggested by the Kalai-Smorodinsky bargaining solution if the risk aversion of one of the players changes. First, we keep the weighting function fixed and vary the utility function; second, we keep the utility function fixed and vary the weighting function. The first case is a generalization to rank dependent utility of known results for expected utility.

Define $\mathbb{B}^{+}=\{B \in \mathbb{B}: s \geq d(B)$ for all $s \in S(B)\}$.

\subsection{Utility risk aversion}

Theorem 3.1 Let $B$ and $C$ be bargaining problems in $\mathbb{B}^{+}$that are identical except that player 2 is more utility risk averse in $C$ than in $B$. Then $K S_{1}(B) \leq K S_{1}(C)$.

The theorem says that a player does not lose when bargaining with a (new) more utility risk averse opponent. Figure 1(a) illustrates this. In the picture we use the fact that under rank dependent utility, the utility function is unique up to scale and location and the Kalai-Smorodinsky solution satisfies scale invariance (see Section 4), so player 2's utility function in bargaining problem $C$ can be scaled such that $d(B)=d(C)$ and $u(B)=u(C)$. 


\subsection{Probabilistic risk aversion}

Theorem 3.2 Let $B$ and $C$ be bargaining problems in $\mathbb{B}^{+}$that are identical except that player 2 is more probabilistically risk averse in $C$ than in $B$. Then $K S_{1}(B) \geq K S_{1}(C)$.

Theorem 3.2 says that a player does not profit from bargaining with a (new) more probabilistically risk averse opponent. This is illustrated in Figure 1(b), where the utility functions of players 2 in $B$ and $C$ are taken to be equal (cf. Lemma 2.2, part 2, and recall that KS is scale invariant).

Observe that both in Theorem 3.1 and in Theorem 3.2 the players 2 in $B$ and $C$ are different players, so that neither these results nor the associated diagrams imply anything about gains or losses of these players. See also the discussion below.

\subsection{Discussion and strategic implications}

Although utility risk aversion and probabilistic risk aversion have a similar impact on the preferences of a decision maker, namely, the more utility or probabilistically risk averse a player is, the less lotteries are preferred over each sure alternative, they have contrary effects in bargaining problems with respect to the Kalai-Smorodinsky bargaining solution. An intuition for this is as follows. If a player can choose between a more and a less utility risk averse opponent, it is advisable to choose the more utility risk averse one, because the marginal utility of the more utility risk averse player diminishes faster, so that this player is easier to satisfy. But if a player can choose between a more and a less probabilistically risk averse opponent, it is advisable to choose the less probabilistically risk adverse one, because the more probabilistically risk averse player weighs probabilities for alternatives with high outcomes lower and therefore asks for more security for obtaining high outcomes.

These considerations represent a positive view: they are of interest for the case in which the Kalai-Smorodinsky solution offers a good description of the bargaining outcome. Additionally, it is interesting to consider the strategic implications of Theorems 3.1 and 3.2 for a bargainer under the assumption that preferences are private knowledge, so that it is possible to be dishonest about the true preferences. These implications are relevant from a normative point of view, in particular, for the question of mechanism design.

Let $B=\left(A, \bar{a}, R D U_{1}, R D U_{2}\right)$ be a bargaining problem, where $R D U_{2}$ corresponds to the true preferences of player 2. We consider four cases, namely that player 2 pretends to be more or less utility or probabilistically risk averse. For simplicity, it is assumed for the rest of this subsection 
that $A$ is finite. This ensures that the image of $L(A)$ in $\mathbb{R}^{2}$ is closed and, therefore, it also ensures the existence of a lottery with rank dependent utility image equal to the point suggested by the Kalai-Smorodinsky solution. Nevertheless, analogous results can be derived for infinite $A$.

1. Does it make sense for player 2 to pretend to be more utility risk averse, with preferences represented by $\widetilde{R D U}_{2}$ ? Let $C=\left(A, \bar{a}, R D U_{1}, \widetilde{R D U}_{2}\right)$. We assume that $\widetilde{R D U}_{2}$ is scaled such that $d(B)=d(C)$ and $u(B)=u(C)$. Let $P, Q \in L(A)$ such that $K S(B)=\left(R D U_{1}(P), R D U_{2}(P)\right)$ and $K S(C)=\left(R D U_{1}(Q), \widetilde{R D U}_{2}(Q)\right)$. Theorem 3.1 implies that $R D U_{1}(P) \leq R D U_{1}(Q)$. If $R D U_{1}(P)<$ $R D U_{1}(Q)$, then Pareto optimality implies $R D U_{2}(P)>R D U_{2}(Q)$. If $R D U_{1}(P)=R D U_{1}(Q)$, then $R D U_{2}(P)=\widetilde{R D U}_{2}(Q)$, because $d(B)=d(C)$ and $u(B)=u(C)$. But $\widetilde{R D U}_{2}(Q) \geq R D U_{2}(Q)$ by Lemma 2.2. Hence, in every case $R D U_{2}(P) \geq R D U_{2}(Q)$. Thus, player 2 never profits from pretending to be more utility risk averse.

2. Does it make sense for player 2 to pretend to be less utility risk averse, with preferences represented by $\widehat{R D U}_{2}$ ? Nothing can be said in general here, as the following example shows.

Example 3.3 Let $A=\{(0,0),(1,0),(0,1),(0.55,0.55)\}, \bar{A}=\{(0,0),(1,0),(0,1),(0.5,0.9))\}, \bar{a}=$ $(0,0), U_{i}(a)=a_{i}$ for $i \in\{1,2\}, \widehat{U}_{2}(0)=0, \widehat{U}_{2}(0.55)=0.2, \widehat{U}_{2}(0.9)=0.55, \widehat{U}_{2}(1)=1$, and $w_{1}(p)=$ $w_{2}(p)=\widehat{w}_{2}(p)=p$ for all $p \in[0,1]$. Let $B=\left(A, \bar{a}, R D U_{1}, R D U_{2}\right)$ and $C=\left(A, \bar{a}, R D U_{1}, \widehat{R D U}_{2}\right)$. Moreover, let $\bar{B}=\left(\bar{A}, \bar{a}, R D U_{1}, R D U_{2}\right)$ and $\bar{C}=\left(\bar{A}, \bar{a}, R D U_{1}, \widehat{R D U}_{2}\right)$. The lotteries that give rise to the Kalai-Smorodinsky solution are, respectively

$$
\begin{aligned}
P & =(1,(0.55,0.55)), \\
Q & =\left(\frac{1}{2},(1,0) ; \frac{1}{2},(0,1)\right), \\
\bar{P} & =\left(\frac{2}{7},(1,0) ; \frac{5}{7},(0.5,0.9)\right), \\
\bar{Q} & =(1 ;(0.55,0.55)) .
\end{aligned}
$$

We have

$$
R D U_{2}(P)=0.55>0.5=R D U_{2}(Q),
$$

but

$$
R D U_{2}(\bar{P})=\frac{9}{14}<0.55=R D U_{2}(\bar{Q}) .
$$

Hence, pretending to be less utility risk averse can be advantagous (player 2 in the pair $\bar{B}, \bar{C}$ ) but also disadvantagous (player 2 in the pair $B, C$ ). 
3. Does it make sense for player 2 to pretend to be less probabilistically risk averse with preferences represented by $\widehat{R D U}_{2}$ ? Let $C=\left(A, \bar{a}, R D U_{1}, \widehat{R D U}_{2}\right)$, and assume again that the utility function $\widehat{U}_{2}$ of player 2 is scaled such that $d(C)=d(B)$ and $u(C)=u(B)$, i.e., $\widehat{U}_{2}=U_{2}$. Let $P, Q \in L(A)$ such that $K S(B)=\left(R D U_{1}(P), R D U_{2}(P)\right)$ and $K S(C)=\left(R D U_{1}(Q), \widehat{R D U}_{2}(Q)\right)$. Theorem 3.2 implies that $R D U_{1}(P) \leq R D U_{1}(Q)$. If $R D U_{1}(P)<R D U_{1}(Q)$, then Pareto optimality implies $R D U_{2}(P)>R D U_{2}(Q)$. If $R D U_{1}(P)=R D U_{1}(Q)$, then $R D U_{2}(P)=\widehat{R D U}_{2}(Q)$, because $d(C)=d(B)$ and $u(C)=u(B)$. But $\widehat{R D U}_{2}(Q) \geq R D U_{2}(Q)$ by Lemma 2.2. Hence, in every case $R D U_{2}(P) \geq R D U_{2}(Q)$. Thus, player 2 never profits from pretending to be less probabilistically risk averse.

4. Does it make sense for player 2 to pretend to be more probabilistically risk averse, with preferences represented by $\widetilde{R D U}_{2}$ ? Nothing can be said in general here, as the following example shows.

Example 3.4 Let $A=\{(0,0),(1,0),(0,1)\}, \bar{A}=A \cup\{(0.55,0.55)\}, \bar{a}=(0,0), U_{i}(a)=a_{i}$ for all $a \in \bar{A}, i \in\{1,2\}, w_{1}(p)=p, w_{2}(p)=\sqrt{p}, \widetilde{w}_{2}(p)=p$ for all $p \in[0,1]$. Let $B=\left(A, \bar{a}, R D U_{1}, R D U_{2}\right)$ and $C=\left(A, \bar{a}, R D U_{1}, \widetilde{R D U_{2}}\right)$, and let $\bar{B}=\left(\bar{A}, \bar{a}, R D U_{1}, R D U_{2}\right)$ and $\bar{C}=\left(\bar{A}, \bar{a}, R D U_{1}, \widetilde{R D U}_{2}\right)$. The lotteries that give rise to the Kalai-Smorodinsky solution in $B, C, \bar{B}$, and $\bar{C}$, are, respectively,

$$
\begin{aligned}
& P \approx(0.618,(1,0) ; 0.382,(0,1)) \\
& Q=(0.5,(1,0) ; 0.5,(0,1)) \\
& \bar{P} \approx(0.461,(1,0) ; 0.241,(0,1) ; 0.298,(0.55,0.55)) \\
& \bar{Q}=(1,(0.55,0.55)) .
\end{aligned}
$$

We have

$$
R D U_{2}(P) \approx 0.618<0.707 \approx R D U_{2}(Q),
$$

but

$$
R D U_{2}(\bar{P}) \approx 0.625>0.55=R D U_{2}(\bar{Q}) .
$$

Hence, pretending to be more probabilistically risk averse can be advantagous (player 2 in the pair $\bar{B}, \bar{C}$ ) but also disadvantagous (player 2 in the pair $B, C$ ). 


\subsection{Extension from $\mathbb{B}^{+}$to $\mathbb{B}$}

In this subsection the restrictions in Theorem 3.2 on bargaining problems are weakened. Riskless alternatives are permitted that are worse than the disagreement alternative for one or both players. In the following, we only consider the effect of weighting functions. For results concerning utility risk aversion in the expected utility case, see Roth and Rothblum [12].

Unfortunately, Theorem 3.2 cannot be generalized to an arbitrary compact set $A$ of riskless alternatives without any further restrictions, as the following example shows.

Example 3.5 Let $A=\{(0,0),(1,1),(-1,2)\}, \bar{a}=(0,0), U_{i}=a_{i}$ for all $a \in A, i \in\{1,2\}$, $w_{1}(p)=p, w_{2}(p)=\sqrt{p}, \widetilde{w}_{2}(p)=p$ for all $p \in[0,1]$. Let $B=\left(A, \bar{a}, R D U_{1}, R D U_{2}\right)$ and $C=$ $\left(A, \bar{a}, R D U_{1}, \widetilde{R D U}_{2}\right)$. Then

$$
K S(B) \approx(0.724,1.929)
$$

but

$$
K S(C)=\left(\frac{3}{4}, \frac{9}{8}\right) .
$$

Hence player 1 obtains more in the game with the more risk averse player 2.

Nevertheless we can still partially generalize Theorem 3.2.

Theorem 3.6 Let $B$ and $C$ be bargaining problems in $\mathbb{B}$ that are identical except that player 2 is more probabilistically risk averse in $C$ than in $B$. If

$$
\frac{u_{2}(B)-d_{2}(B)}{u_{1}(B)-d_{1}(B)} \leq \frac{u_{2}(C)-d_{2}(C)}{u_{1}(C)-d_{1}(C)}
$$

then $K S_{1}(C) \leq K S_{1}(B)$.

Condition (1) says that the line segment with $d(C)$ and $u(C)$ as endpoints is steeper (has a higher slope) than the line segment with $d(B)$ and $u(B)$ as endpoints. If $d(B)=d(C)$ - which can always be arranged without loss of generality - condition (1) holds in particular if $U_{1}(a) \geq d_{1}(B)$ for all $a \in A$, that is, if player 1 weakly prefers all riskless alternatives to the disagreement alternative.

\section{An axiomatization of the Kalai-Smorodinsky bargaining solution}

The Kalai-Smorodinsky solution for rank dependent utility bargaining problems can be characterized by a similar set of conditions as in the expected utility case (see Kalai and Smorodinsky, [5]). 
The axioms are as follows, formulated for a bargaining solution $F$.

Symmetry For every $(S, d) \in \mathbb{B}$ with (i) if $\left(s_{1}, s_{2}\right) \in S$ then also $\left(s_{2}, s_{1}\right) \in S$; (ii) $d_{1}=d_{2}$, we have $F_{1}(S, d)=F_{2}(S, d)$.

Scale invariance For all $(S, d) \in \mathbb{S}$ and $a, b \in \mathbb{R}^{2}$ with $a>0$, we have $F(a S+b, a d+b)=$ $a F(S, d)+b$, where $a x:=\left(a_{1} x_{1}, a_{2} x_{2}\right)$ for all $x \in \mathbb{R}^{2}$ and $a S+b:=\{a s+b: s \in S\}$.

Weak Pareto optimality For every $(S, d) \in \mathbb{S}$ there is no $x \in S$ with $x>F(S, d)$.

Individual monotonicity For all $(S, d),(\bar{S}, \bar{d}) \in \mathbb{S}$ and all $i \neq j \in\{1,2\}$ with (i) $\bar{S} \subseteq S$; (ii) $\bar{d}=d$; and (iii) $u_{i}(\bar{S}, \bar{d})=u_{i}(S, d)$, we have $F_{j}(\bar{S}, \bar{d}) \leq F_{j}(S, d)$.

Observe that these conditions still make sense in the rank dependent utility setting. In particular, scale invariance reflects the fact that the utility function in rank dependent utility is unique up to a positive linear transformation. Also the following characterization is similar to the one obtained in Kalai and Smorodinsky [5] but in the proof (see the Appendix) the construction of auxiliary bargaining problems needs special attention because of the different domain, in particular the presence and construction of nonconvex problems.

Theorem 4.1 Let $F$ be a bargaining solution. Then $F$ satisfies symmetry, scale invariance, weak Pareto optimality and individual monotonicity if, and only if, $F=K S$.

\section{Conclusion}

It has been shown in this paper that, with respect to the Kalai-Smorodinsky solution, in many situations it is on the one hand not advantagous to bargain against a more probabilistically risk averse opponent, but on the other hand also not advantagous to bargain against a less utility risk averse opponent. These results are surprising, since both probabilistic and utility risk aversion arise from the same definition of risk aversion. The intuition behind the results could be that a more utility risk averse bargainer is easier to satisfy and is therefore less demanding in a bargaining problem, whereas a more probabilistically risk averse bargainer wants a higher guarantee to obtain good outcomes and is therefore more demanding. In this paper we varied the attitude towards risk either with respect to the weighting function or with respect to the utility function, and kept the 
other component fixed. It may be interesting to design an experiment to test which of the two components has more influence in bargaining problems.

The main results can be generalized to $n$-person bargaining problems with the restriction that the feasible sets are made comprehensive. It is also possible to adapt the results to bargaining solutions exhibiting certain monotonicity properties. For instance, the egalitarian bargaining solution defined on the set of comprehensive problems or the Kalai-Rosenthal solution (Kalai and Rosenthal, [6]) are such candidates.

The extension of the Nash bargaining solution (Nash, [9]) to bargaining problems under rank dependent utility is more problematic. In particular, feasible sets are not necessarily convex, so that the Nash bargaining solution is not uniquely defined. Nevertheless, even if we restrict attention to bargaining problems where the Nash bargaining solution is well defined, it does not behave regularly. Both cases can arise: a player can gain or lose with respect to the Nash bargaining solution when bargaining with a more probabilistically risk averse opponent.

Cumulative prospect theory (Tversky and Kahneman, [19]) differs from rank dependent utility in the sense that probabilities are weighted in a different way, depending on whether the associated riskless alternatives are preferred to a specific reference point or not. All of the above results can be generalized to cumulative prospect theory. There, a distinction between gains and losses is made, and a third factor influences the risk attitude of a decision maker, namely loss aversion. The impact of loss aversion in bargaining problems is a subject for further research. Also Shalev [16] considers loss aversion in bargaining but in a quite different framework.

\section{A Appendix: Proofs}

Before proving Lemmas 2.3 and 2.4 we first prove some auxiliary results from which these lemmas will be derived. By $\|\cdot\|$ we denote the Euclidean norm on $\mathbb{R}^{2}$. We say that a feasible set $S$ has a hole if there is an $h \in \mathbb{R}^{2}$ and an $\varepsilon>0$ such that (i) $x \notin S$ for all $x \in \mathbb{R}^{2}$ with $\|x-h\|<\varepsilon$ and (ii) for every $x \in \mathbb{R}^{2}$ with $x \neq 0$ there is a number $\lambda_{x}>0$ with $h+\lambda_{x} x \in S$.

Lemma A.1 Let $B=\left(A, \bar{a}, R D U_{1}, R D U_{2}\right) \in \mathbb{B}$. Then $S(B)$ contains no holes.

Proof. Define $T=\left\{\left(R D U_{1}(P), R D U_{2}(P)\right): P \in L(A)\right\}$. By definition, $S(B)=c l\{T\}$. We say that $T$ contains an almost-hole if there exists an $h \in \mathbb{R}^{2}$ and a $\varepsilon>0$ such that (i) $x \notin S$ for all $x \in \mathbb{R}^{2}$ with $\|x-h\|<\varepsilon$ and (ii) there is a $y \in \mathbb{R}^{2}$ such that for every $x \in \mathbb{R}^{2}$ with $x \neq 0$ and 
$x \neq \tau y$ for every number $\tau \geq 0$ there is a number $\lambda_{x}>0$ with $h+\lambda_{x} x \in S$. Since $T$ is connected and $S(B)$ is the closure of $T$, it is sufficient to show that $T$ contains no almost-holes. We first prove the following claim.

Claim. For all $s, t \in T$ with either $s_{1}=t_{1}$ or $s_{2}=t_{2}$ the straight line segment between these two points is contained in $T$, i.e., $[s, t] \subseteq T$.

Proof of the Claim: Let $s, t \in T$ with $s_{2}=t_{2}$ (the case $s_{1}=t_{1}$ is analogous). Let $P=$ $\left(p^{1}, a^{1} ; \ldots ; p^{k}, a^{k}\right)$ and $Q=\left(q^{1}, b^{1} ; \ldots ; q^{n}, b^{n}\right) \in L(A)$ with $\left(R D U_{1}(P), R D U_{2}(P)\right)=s$ and

$\left(R D U_{1}(Q), R D U_{2}(Q)\right)=t$. Without loss of generality assume that all probabilities in $P$ and $Q$ are positive, and let $C=\left\{a^{1}, \ldots, a^{k}, b^{1}, \ldots, b^{n}\right\}$. Let $U_{2}$ be the utility function of player 2 .

Case 1: For all $c \in C, U_{2}(c)=s_{2}$. Then all lotteries on elements of $C$ have rank dependent utility equal to $s_{2}$ for player 2 and all points in $[s, t]$ can be obtained by such lotteries. Hence, $[s, t] \subseteq T$.

Case 2: There are $c, \widetilde{c} \in C$ with $U_{2}(c) \neq U_{2}(\widetilde{c})$. Let $\bar{c} \in \arg \max \left\{U_{2}(c): c \in C\right\}$ and $\underline{c} \in$ $\arg \min \left\{U_{2}(c): c \in C\right\}$. Clearly, $U_{2}(\bar{c})>s_{2}>U_{2}(\underline{c})$. Let $R=(\rho, \bar{c} ; 1-\rho, \underline{c})$ be the unique lottery with $R D U_{2}(R)=s_{2}$. Let $r=\left(R D U_{1}(R), R D U_{2}(R)\right)$, then we will show that the straight line segment between $s$ and $r$ is in $T$. Similarly, it can be shown that the straight line segment between $r$ and $t$ is in $T$. Therefore, the straight line segment between $s$ and $t$ is contained in $T$.

Observe that for every lottery $\widetilde{P}=\left(\widetilde{p}^{1}, a^{1} ; \ldots ; \widetilde{p}^{k}, a^{k} ; \widetilde{p}^{k+1}, \bar{c} ; \widetilde{p}^{k+2}, \underline{c}\right)$ with $R D U_{2}(\widetilde{P})=s_{2}$, for every $i \in\{1, \ldots, k\}$ and for every $\varepsilon$ with $0 \leq \varepsilon \leq \widetilde{p}^{i}$, there exist some $\lambda, \pi \geq 0$ with $\lambda+\pi=\varepsilon$ such that for the lottery $\widetilde{P^{\varepsilon}}=\left(\widetilde{p}^{1}, a^{1} ; \ldots ; \widetilde{p}^{i-1}, a^{i-1} ; \widetilde{p}^{i}-\varepsilon, a^{i} ; \widetilde{p}^{i+1}, a^{i+1} ; \ldots ; \widetilde{p}^{k}, a^{k} ; \widetilde{p}^{k+1}+\lambda, \bar{c} ; \widetilde{p}^{k+2}+\pi, \underline{c}\right)$ we have $R D U_{2}\left(\widetilde{P^{\varepsilon}}\right)=s_{2}$.

Starting with $P$ we now gradually construct new lotteries with $R D U_{2}$-value equal to $s_{2}$ by shifting, for every $a^{i} \notin\{\bar{c}, \underline{c}\}$, the corresponding decision weight $p^{i}$ in a continuous way simultanously to $\bar{c}$ and $\underline{c}$ until we reach $R$, such that the $R D U_{2}$-value stays constant. In this way we obtain every point on the straight line segment between $s$ and $r$ as the image of at least one of the contructed lotteries. This completes the proof of the Claim.

We now continue with the proof of the lemma. Assume for contradiction that $T$ contains an almost-hole, and let $h \in \mathbb{R}^{2}$ be as in the definition of an almost-hole. In particular, there exist $\lambda_{(1,0)}, \lambda_{(-1,0)}>0$ with $h+\lambda_{(1,0)}(1,0), h+\lambda_{(-1,0)}(-1,0) \in T$, or there exist $\lambda_{(0,1)}, \lambda_{(0,-1)}>0$ with $h+\lambda_{(0,1)}(0,1), h+\lambda_{(0,-1)}(0,-1) \in T$. Assume the former is the case, the latter is analogous. Observe that $\left(h+\lambda_{(1,0)}(1,0)\right)_{2}=\left(h+\lambda_{(-1,0)}(-1,0)\right)_{2}=h_{2}$. Hence the Claim implies that $[h-$ 
$\left.\lambda_{(-1,0)}(1,0), h+\lambda_{(1,0)}(1,0)\right] \subseteq T$. In particular, $h \in T$, which contradicts (i) in the definition of an almost-hole. Therefore, $T$ contains no almost-holes and the proof of the lemma is complete.

The following lemma is needed to derive Lemma 2.4.

Lemma A.2 Let $B=\left(A, \bar{a}, R D U_{1}, R D U_{2}\right) \in \mathbb{B}$ with utility functions $U_{1}$ and $U_{2}$ and weighting functions $w_{1}$ and $w_{2}$ for players 1 and 2 , respectively. Let $\bar{b}^{2} \in \arg \max \left\{U_{2}(a): a \in A\right\}$ and $\bar{b}^{1} \in \arg \max \left\{U_{1}(a): a \in A\right\}$. Let $S=S(B)$ and $x \in P(S)$. Then

(i) if $x_{2}<U_{2}\left(\bar{b}^{2}\right)$ then for every $\varepsilon>0$ there is a $y \in S$ with $y_{1}<x_{1}, y_{2}>x_{2}$, and $\|x-y\|<\varepsilon$.

(ii) if $x_{1}<U_{1}\left(\bar{b}^{1}\right)$ then for every $\varepsilon>0$ there is a $y \in S$ with $y_{2}<x_{2}, y_{1}>x_{1}$, and $\|x-y\|<\varepsilon$.

Proof. We only prove (i), the proof of (ii) is analogous. Suppose that (i) is not true. Together with $x \in P(S)$ this implies that there is a $\hat{\varepsilon}>0$ such that

$$
S \cap\left\{y \in \mathbb{R}^{2}:\|y-x\|<\hat{\varepsilon}, y_{2}>x_{2}\right\}=\emptyset .
$$

Let $P \in L(A)$ be an arbitrary lottery with $R D U_{2}(P) \leq x_{2}$. Such a lottery is of the form $P=\left(p^{0}, \bar{b}^{2} ; p^{1}, a^{1} ; \ldots ; p^{n}, a^{n}\right)$ with $U_{2}\left(\bar{b}^{2}\right) \geq U_{2}\left(a^{1}\right) \geq \cdots \geq U_{2}\left(a^{n}\right)$, and possibly, $p^{0}=0$.

Claim 1. There is a number $0 \leq \bar{p}<1$, independent of $P$, such that $p^{0} \leq \bar{p}$.

Proof of Claim 1. Let $\underline{b}^{2} \in \arg \min \left\{U_{2}(a): a \in A\right\}$. Since $R D U_{2}(P) \leq x_{2}$ we have

$$
\begin{aligned}
x_{2} & \geq w_{2}\left(p^{0}\right) U_{2}\left(\bar{b}^{2}\right)+\sum_{i=1}^{n}\left[w_{2}\left(p^{0}+\cdots+p^{i}\right)-w_{2}\left(p^{0}+\cdots+p^{i-1}\right)\right] U_{2}\left(a^{i}\right) \\
& \geq w_{2}\left(p^{0}\right) U_{2}\left(\bar{b}^{2}\right)+\sum_{i=1}^{n}\left[w_{2}\left(p^{0}+\cdots+p^{i}\right)-w_{2}\left(p^{0}+\cdots+p^{i-1}\right)\right] U_{2}\left(\underline{b}^{2}\right) \\
& =w_{2}\left(p^{0}\right)\left[U_{2}\left(\bar{b}^{2}\right)-U_{2}\left(\underline{b}^{2}\right)\right]+U_{2}\left(\underline{b}^{2}\right) .
\end{aligned}
$$

Hence

$$
w_{2}\left(p^{0}\right) \leq \frac{x_{2}-U_{2}\left(\underline{b}^{2}\right)}{U_{2}\left(\bar{b}^{2}\right)-U_{2}\left(\underline{b}^{2}\right)}<1
$$

so that

$$
p^{0} \leq w_{2}^{-1}\left(\frac{x_{2}-U_{2}\left(\underline{b}^{2}\right)}{U_{2}\left(\bar{b}^{2}\right)-U_{2}\left(\underline{b}^{2}\right)}\right):=\bar{p}<1 .
$$

This proves the claim. 
Claim 2. Let $m=\frac{1}{2}\left(x_{2}+U_{2}\left(\bar{b}^{2}\right)\right)$, and let $k \in\{1, \ldots, n\}$ such that $U_{2}\left(a^{i}\right)<m \Leftrightarrow i \geq k$. Then there is a number $0<\underline{p} \leq 1$, independent of $P$, such that $\sum_{i=k}^{n} p^{i} \geq \underline{p}$.

Proof of Claim 2. Since $\operatorname{RDU}_{2}(P) \leq x_{2}$ we have

$$
\begin{aligned}
x_{2} \geq & w_{2}\left(p^{0}\right) U_{2}\left(\bar{b}^{2}\right)+\sum_{i=1}^{n}\left[w_{2}\left(p^{0}+\cdots+p^{i}\right)-w_{2}\left(p^{0}+\cdots+p^{i-1}\right)\right] U_{2}\left(a^{i}\right) \\
\geq & w_{2}\left(p^{0}\right) U_{2}\left(\bar{b}^{2}\right)+\sum_{i=1}^{k-1}\left[w_{2}\left(p^{0}+\cdots+p^{i}\right)-w_{2}\left(p^{0}+\cdots+p^{i-1}\right)\right] m \\
& +\sum_{i=k}^{n}\left[w_{2}\left(p^{0}+\cdots+p^{i}\right)-w_{2}\left(p^{0}+\cdots+p^{i-1}\right)\right] U_{2}\left(\underline{b}^{2}\right) \\
= & w_{2}\left(p^{0}\right) U_{2}\left(\bar{b}^{2}\right)-w_{2}\left(p^{0}\right) m+w\left(p^{0}+\cdots+p^{k-1}\right)\left[m-U_{2}\left(\underline{b}^{2}\right)\right]+U_{2}\left(\underline{b}^{2}\right) \\
\geq & w_{2}\left(p^{0}+\cdots+p^{k-1}\right)\left[m-U_{2}\left(\underline{b}^{2}\right)\right]+U_{2}\left(\underline{b}^{2}\right) .
\end{aligned}
$$

Hence

$$
w_{2}\left(p^{0}+\cdots+p^{k-1}\right) \leq \frac{x_{2}-U_{2}\left(\underline{b}^{2}\right)}{m-U_{2}\left(\underline{b}^{2}\right)}<1,
$$

so that

$$
p^{0}+\cdots+p^{k-1} \leq w_{2}^{-1}\left(\frac{x_{2}-U_{2}\left(\underline{b}^{2}\right)}{m-U_{2}\left(\underline{b}^{2}\right)}\right)<1
$$

and therefore

$$
\sum_{i=k}^{n} p^{i} \geq 1-w_{2}^{-1}\left(\frac{x_{2}-U_{2}\left(\underline{b}^{2}\right)}{m-U_{2}\left(\underline{b}^{2}\right)}\right):=\underline{p}>0 .
$$

This proves the claim.

Claim 3. Let $\bar{p}$ and $\underline{p}$ be as in Claims 1 and 2 , and let $0<\varepsilon \leq \min \{1-\bar{p}, \underline{p}\}$. Let $m$ and $k$ be as in Claim 2 and let $P^{\varepsilon}$ be a lottery obtained from $P$ by increasing $p^{0}$ with $\varepsilon$ and weakly decreasing, for $i=k, \ldots, n, p^{i}$ with $\varepsilon^{i} \geq 0$ such that $\sum_{i=k}^{n} \varepsilon^{i}=\varepsilon$. Then there is a number $0<r(\varepsilon) \in \mathbb{R}$, independent of $P$, such that $R D U_{2}\left(P^{\varepsilon}\right) \geq R D U_{2}(P)+r(\varepsilon)$.

Proof of Claim 3. Define $\underline{w}(\varepsilon):=\min \left\{w_{2}(q+\varepsilon)-w_{2}(q): 0 \leq q \leq 1-\varepsilon\right\}$. Observe that $\underline{w}(\varepsilon)>0$. 
By rearranging the expression in the definition of RDU and setting $a^{0}:=\bar{b}^{2}$, we have:

$$
\begin{aligned}
R D U_{2}\left(P^{\varepsilon}\right)= & \sum_{i=0}^{k-1} w_{2}\left(\left(p^{0}+\varepsilon\right)+p^{1}+\cdots+p^{i}\right)\left[U_{2}\left(a^{i}\right)-U_{2}\left(a^{i+1}\right)\right] \\
& +\sum_{i=k}^{n-1} w_{2}\left(\left(p^{0}+\varepsilon\right)+p^{1}+\cdots+p^{k-1}+\left(p^{k}-\varepsilon^{k}\right)+\cdots+\left(p^{i}-\varepsilon^{i}\right)\right)\left[U_{2}\left(a^{i}\right)-U_{2}\left(a^{i+1}\right)\right] \\
& +U_{2}\left(a^{n}\right) \\
\geq & \sum_{i=0}^{k-1} w_{2}\left(\left(p^{0}+\varepsilon\right)+p^{1}+\cdots+p^{i}\right)\left[U_{2}\left(a^{i}\right)-U_{2}\left(a^{i+1}\right)\right] \\
& +\sum_{i=k}^{n-1} w_{2}\left(p^{0}+p^{1}+\cdots+p^{i}\right)\left[U_{2}\left(a^{i}\right)-U_{2}\left(a^{i+1}\right)\right]+U_{2}\left(a^{n}\right) \\
\geq & \sum_{i=0}^{k-1}\left[w_{2}\left(p^{0}+p^{1}+\cdots+p^{i}\right)+\underline{w}(\varepsilon)\right]\left[U_{2}\left(a^{i}\right)-U_{2}\left(a^{i+1}\right)\right] \\
& +\sum_{i=k}^{n-1} w_{2}\left(p^{0}+p^{1}+\cdots+p^{i}\right)\left[U_{2}\left(a^{i}\right)-U_{2}\left(a^{i+1}\right)\right]+U_{2}\left(a^{n}\right) \\
= & R D U_{2}(P)+\underline{w}(\varepsilon)\left[U_{2}\left(a^{0}\right)-U_{2}\left(a^{k}\right)\right] \\
\geq & R D U_{2}(P)+\underline{w}(\varepsilon)\left[U_{2}\left(\bar{b}^{2}\right)-m\right] .
\end{aligned}
$$

The claim follows by defining $r(\varepsilon):=\underline{w}(\varepsilon)\left[U_{2}\left(\bar{b}^{2}\right)-m\right]$.

For the next claim, let $\underline{b}^{1} \in \arg \min \left\{U_{1}(a): a \in A\right\}$.

Claim 4. Let $\eta>0$ and let $Q=\left(q^{1}, a^{1} ; \ldots ; q^{n}, a^{n}\right)$ and $Q^{\eta}=\left(\tilde{q}^{1}, a^{1} ; \ldots ; \tilde{q}^{n}, a^{n}\right)$ be arbitrary lotteries such that $Q^{\eta}$ arises from $Q$ by shifting around probability such that $\sum_{i=1}^{n}\left|q^{i}-\tilde{q}^{i}\right| \leq \eta$. Then there are positive numbers $M_{1}(\eta)$ and $M_{2}(\eta)$, independent of $Q$, such that $\left|R D U_{1}(Q)-R D U_{1}\left(Q^{\eta}\right)\right| \leq M_{1}(\eta)$ and $\left|R D U_{2}(Q)-R D U_{2}\left(Q^{\eta}\right)\right| \leq M_{2}(\eta)$, and, moreover, $M_{1}(\eta), M_{2}(\eta) \rightarrow 0$ as $\eta \rightarrow 0$.

Proof of Claim 4. We only prove the first inequality, the proof of the second one is analogous. Without loss of generality assume $U_{1}\left(a^{1}\right) \geq \cdots \geq U\left(a^{n}\right)$. Then

$$
\begin{aligned}
\left|R D U_{1}(Q)-R D U_{1}\left(Q^{\eta}\right)\right|= & \mid \sum_{i=1}^{n-1} w_{1}\left(q^{1}+\cdots+q^{i}\right)\left[U_{1}\left(a^{i}\right)-U_{1}\left(a^{i+1}\right)\right]+U_{1}\left(a^{n}\right) \\
& -\sum_{i=1}^{n-1} w_{1}\left(\tilde{q}^{1}+\cdots+\tilde{q}^{i}\right)\left[U_{1}\left(a^{i}\right)-U_{1}\left(a^{i+1}\right)\right]-U_{1}\left(a^{n}\right) \mid \\
= & \left|\sum_{i=1}^{n-1}\left[w_{1}\left(q^{1}+\cdots+q^{i}\right)-w_{1}\left(\tilde{q}^{1}+\cdots+\tilde{q}^{i}\right)\right]\left[U_{1}\left(a^{i}\right)-U_{1}\left(a^{i+1}\right)\right]\right| \\
\leq & \sum_{i=1}^{n-1} \max \left\{\left|w_{1}(q+\eta)-w(q)\right|: 0 \leq q \leq 1-\eta\right\}\left[U_{1}\left(a^{i}\right)-U_{1}\left(a^{i+1}\right)\right] \\
\leq & M_{1}(\eta),
\end{aligned}
$$


with $M_{1}(\eta):=\max \left\{\left|w_{1}(q+\eta)-w(q)\right|: 0 \leq q \leq 1-\eta\right\}\left[U_{1}\left(\bar{b}^{1}\right)-U_{1}\left(\underline{b}^{1}\right)\right]>0$. Since $M_{1}(\eta) \rightarrow 0$ as $\eta \rightarrow 0$ the proof of Claim 4 is complete.

We will now complete the proof of the lemma. Take a sequence of lotteries $P_{1}, P_{2}, \ldots$ with $\left(R D U_{1}\left(P_{\ell}\right), R D U_{2}\left(P_{\ell}\right)\right) \rightarrow x$ as $\ell \rightarrow \infty$. Assume without loss of generality that $\left\|\left(R D U_{1}\left(P_{\ell}\right), R D U_{2}\left(P_{\ell}\right)\right)-x\right\|<\frac{\hat{\varepsilon}}{4}$ for every $\ell$. By $(2), R D U_{2}\left(P_{\ell}\right) \leq x_{2}$ for every $\ell$. Take $\eta>0$ so small that $M_{1}(\eta), M_{2}(\eta)<\frac{\hat{\varepsilon}}{4}\left(\right.$ with $M_{1}(\eta), M_{2}(\eta)$ as in Claim 4.). Finally, take $\varepsilon>0$ with $2 \varepsilon<\eta$ and $0<\varepsilon \leq \min \{1-\bar{p}, \underline{p}\}$ (as in Claim 3). Then Claim 3 implies that for an $\ell^{\prime}$ sufficiently large we have $R D U_{2}\left(P_{\ell^{\prime}}^{\varepsilon}\right) \geq R D U_{2}\left(P_{\ell^{\prime}}\right)+r(\varepsilon)>x_{2}$, with $P_{\ell^{\prime}}^{\varepsilon}$ and $r(\varepsilon)$ as in Claim 3. Since $2 \varepsilon<\eta$ it follows by Claim 4 that $\left|R D U_{1}\left(P_{\ell^{\prime}}\right)-R D U_{1}\left(P_{\ell^{\prime}}^{\varepsilon}\right)\right| \leq M_{1}(\eta)$ and $\left|R D U_{2}\left(P_{\ell^{\prime}}\right)-R D U_{2}\left(P_{\ell^{\prime}}^{\varepsilon}\right)\right| \leq M_{2}(\eta)$. Hence, $\left|R D U_{1}\left(P_{\ell^{\prime}}\right)-R D U_{1}\left(P_{\ell^{\prime}}^{\varepsilon}\right)\right| \leq \frac{\hat{\varepsilon}}{4}$ and $\left|R D U_{2}\left(P_{\ell^{\prime}}\right)-R D U_{2}\left(P_{\ell^{\prime}}^{\varepsilon}\right)\right| \leq \frac{\hat{\varepsilon}}{4}$. Thus, ||$\left(R D U_{1}\left(P_{\ell^{\prime}}^{\varepsilon}\right), R D U_{2}\left(P_{\ell^{\prime}}^{\varepsilon}\right)\right)-$ $x \|<\hat{\varepsilon}$ whereas $R D U_{2}\left(P_{\ell^{\prime}}^{\varepsilon}\right)>x_{2}$, contradicting (2). This completes the proof of lemma.

Proof of Lemma 2.4. Let $\bar{x}$ be the point of $S$ with maximal first coordinate among the points of $S$ with maximal second coordinate. Similarly, let $\underline{x}$ be the point of $S$ with maximal second coordinate among the points of $S$ with maximal first coordinate. Clearly, $\bar{x}$ and $\underline{x}$ are Pareto optimal points, and for every point $x \in P(S)$ we have $\bar{x}_{1} \leq x_{1} \leq \underline{x}_{1}$.

To prove the lemma it is sufficient to prove that for every $\alpha \in \mathbb{R}$ with $\bar{x}_{1} \leq \alpha \leq \underline{x}_{1}$ there is a number $f(\alpha) \in \mathbb{R}$ with $(\alpha, f(\alpha)) \in P(S)$ : such a number must then be unique and the implied function $f$ is strictly decreasing and, by Lemma A.2, also continuous. Hence, $f$ satisfies the requirements of the lemma.

For $\alpha=\bar{x}_{1}$ take $f(\alpha)=\bar{x}_{2}$ and for $\alpha=\underline{x}_{1}$ take $f(\alpha)=\underline{x}_{2}$. For $\alpha \in \mathbb{R}$ with $\bar{x}_{1}<\alpha<\underline{x}_{1}$ define $f(\alpha):=\max \{\beta:(\alpha, \beta) \in S\}$. Suppose $(\alpha, f(\alpha)) \notin P(S)$. Then let $x$ be the point with maximal first coordinate among all points of $D:=\{y \in S: y \geq(\alpha, f(\alpha))\}$ with maximal second coordinate. Clearly, $x \in P(S), x_{1}>\alpha$, and $x_{2}<\bar{x}_{2}$. Then Lemma A.2(i) implies that there is a point $y \in D$ with $y_{2}>x_{2}$, a contradiction to the definition of $x$. This completes the proof of the lemma.

For $(S, d) \in \mathbb{S}$ we define $P^{++}(S)=\left\{s \in S:\right.$ there exists no $t \in S$ with $t_{1} \geq s_{1}, t_{2} \geq s_{2}$ and $\left.t \neq s\right\}$, $P^{+-}(S)=\left\{s \in S:\right.$ there exists no $t \in S$ with $t_{1} \geq s_{1}, t_{2} \leq s_{2}$ and $\left.t \neq s\right\}, P^{-+}(S)=\{s \in S:$ there exists no $t \in S$ with $t_{1} \leq s_{1}, t_{2} \geq s_{2}$ and $\left.t \neq s\right\}$, and $P^{--}(S)=\left\{s \in S:\right.$ there exists no $t \in S$ with $t_{1} \leq s_{1}, t_{2} \leq s_{2}$ and $\left.t \neq s\right\}$. Observe that $P^{++}(S)=$ $P(S)$.

The following lemma is a generalization of Lemma 2.4. The proof can proceed completely analogous to the proof of Lemma 2.4, and is therefore omitted. The lemma is used in the proof of 
Lemma 2.3.

Lemma A.3 Let $(S, d) \in \mathbb{S}$. Then there are closed intervals $I^{++}, I^{+-}, I^{-+}$, and $I^{--} \subseteq \mathbb{R}$, continuous strictly decreasing functions $f^{++}: I^{++} \rightarrow \mathbb{R}, f^{--}: I^{--} \rightarrow \mathbb{R}$ and continuous strictly increasing functions $f^{+-}: I^{+-} \rightarrow \mathbb{R}, f^{-+}: I^{-+} \rightarrow \mathbb{R}$ such that

$$
\begin{aligned}
& P^{++}(S)=\left\{\left(t, f^{++}(t)\right): t \in I^{++}\right\}, \\
& P^{+-}(S)=\left\{\left(t, f^{+-}(t)\right): t \in I^{+-}\right\}, \\
& P^{-+}(S)=\left\{\left(t, f^{-+}(t)\right): t \in I^{-+}\right\}, \\
& P^{--}(S)=\left\{\left(t, f^{--}(t)\right): t \in I^{--}\right\} .
\end{aligned}
$$

Proof of Lemma 2.3. We first observe that the points of $S$ with maximal second coordinates form a straight line segment, possibly consisting of only one point: all these points can be obtained by lotteries between riskless alternatives with minimal and maximal utility for player 1 among all the riskless alternatives that have maximal utility for player 2. The same is true for all points with minimal second coordinates, or maximal or minimal first coordinates.

Let $s, t \in S$ be points that have one coordinate in common. Without loss of generality assume $s_{1}<t_{1}$ and $s_{2}=t_{2}$. If $s_{2}$ is maximal or minimal in $S$ then $[s, t] \subseteq S$ follows from the observations in preceding paragraph. Otherwise, any point $h \in[s, t]$ strictly between $s$ and $t$ satisfies condition (ii) in the definition of a hole: this follows from the observations in the preceding paragraph and from Lemma A.3. Suppose that there would be such an $h$ with $h \notin S$. Since $S$ is closed there must be an $\varepsilon$-neigborhood of $h$ that is not in $S$. Hence, $h$ satisfies also condition (i) in the definition of a hole, and thus $S$ has a hole. This contradicts Lemma A.1 and completes the proof.

Proof of Lemma 2.5. Let $I=[\alpha, \beta]$. If $\alpha \neq \beta$, define the continuous strictly increasing function $w:[0,1] \rightarrow[0,1]$ by

$$
w\left(\frac{\beta-t}{\beta-\alpha}\right)=\frac{f(t)-f(\beta)}{f(\alpha)-f(\beta)}
$$

for every $t \in I$. In particular, $w(0)=0$ and $w(1)=1$, so that $w$ satisfies all the requirements of a weighting function.

If $\alpha=\beta$, then for $\bar{a}$ take any point in $\mathbb{R}^{2}$ with $\bar{a}<(\alpha, f(\alpha))$.

Define $A=\left\{\bar{a},\left(\alpha, \bar{a}_{2}\right),(\alpha, f(\alpha)),\left(\bar{a}_{1}, f(\alpha)\right)\right\}$.

If $\alpha \neq \beta$ then take $\bar{a}=(\alpha, f(\beta))$. Define $A=\{\bar{a},(\beta, f(\beta)),(\alpha, f(\alpha))\}$. 
Define $U_{i}(a)=a_{i}$ for all $a \in A$ and $i \in\{1,2\}, w_{1}(p)=p$ and $w_{2}(p)=w(p)$ for all $p \in[0,1]$. Let $B=\left(A, \bar{a}, R D U_{1}, R D U_{2}\right)$. By construction,

$\{(t, f(t)): t \in I\}=\left\{\left(R D U_{1}(P), R D U_{2}(P)\right): P=((\alpha, f(\alpha)), p ;(\beta, f(\beta)), 1-p)\right.$ for some $\left.p \in[0,1].\right\}$

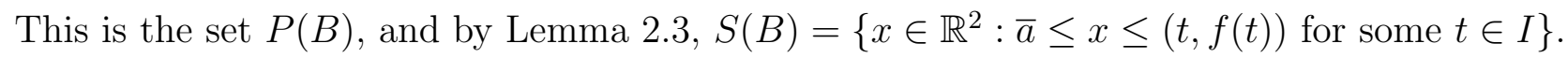

Proof of Theorem 3.1. Let $B=\left(A, \bar{a}, R D U_{1}, R D U_{2}\right), C=\left(A, \bar{a}, R D U_{1}, \widetilde{R D U}_{2}\right) \in \mathbb{B}^{+}$such that $R D U_{2}$ is associated with $U_{2}$ and $w_{2}$ and $\widetilde{R D U}_{2}$ with $\widetilde{U}_{2}=k \circ U_{2}$ and $w_{2}$ for a concave strictly increasing transformation $k: \mathbb{R} \rightarrow \mathbb{R}$. It can be assumed that $\widetilde{U_{2}}$ is scaled such that $d(B)=d(C)$ and $u(B)=u(C)$, since the Kalai-Smorodinsky solution is scale invariant (see Theorem 4.1). This implies that $\widetilde{U}_{2}(a) \geq U_{2}(a)$ for all $a \in A$ and therefore,

$$
\widetilde{R D U_{2}}(P) \geq R D U_{2}(P)
$$

for all $P \in L(A)$. Consider $K S(B)$. Because $d(B)=d(C)$ and $u(B)=u(C)$, we either have $K S(B) \leq K S(C)$ or $K S(B)>K S(C)$. Weak Pareto optimality of the Kalai-Smorodinsky solution (see Theorem 4.1) and (3) imply $K S(B) \leq K S(C)$, hence $K S_{1}(B) \leq K S_{1}(C)$.

Proof of Theorem 3.2. Let $B=\left(A, \bar{a}, R D U_{1}, R D U_{2}\right), C=\left(A, \bar{a}, R D U_{1}, \widetilde{R D U}_{2}\right) \in \mathbb{B}^{+}$such that $R D U_{2}$ is associated with $U_{2}$ and $w_{2}$ and $\widetilde{R D U}_{2}$ with $U_{2}$ and $\widetilde{w}_{2}$ where $w_{2}(p) \geq \widetilde{w}_{2}(p)$ for all $p \in[0,1]$. (Hence, without loss of generality we have scaled the utility function of player 2 in $C$ such that it is equal to $U_{2}$, cf. Lemma 2.2.) Then by Lemma 2.2, part 2,

$$
R D U_{2}(P) \geq \widetilde{R D U}_{2}(P)
$$

for all $P \in L(A)$. Consider $K S(C)$. Because $d(B)=d(C)$ and $u(B)=u(C)$, either $K S(C) \leq$ $K S(B)$ or $K S(C)>K S(B)$. Weak Pareto optimality of the Kalai-Smorodinsky solution (see Theorem 4.1) and (4) imply $K S(C) \leq K S(B)$, hence $K S_{1}(B) \geq K S_{1}(C)$.

Proof of Theorem 3.6. Let $B=\left(A, \bar{a}, R D U_{1}, R D U_{2}\right), C=\left(A, \bar{a}, R D U_{1}, \widetilde{R D U_{2}}\right) \in \mathbb{B}$ such that $R D U_{2}$ is associated with $U_{2}$ and $w_{2}$ and $\widetilde{R D U}_{2}$ with $U_{2}$ and $\widetilde{w}_{2}$ where $w_{2}(p) \geq \widetilde{w}_{2}(p)$ for all $p \in[0,1]$-this is similar as in the proof of Theorem 3.2 -and $\frac{u_{2}(B)-d_{2}(B)}{u_{1}(B)-d_{1}(B)} \leq \frac{u_{2}(C)-d_{2}(C)}{u_{1}(C)-d_{1}(C)}$. Because the Kalai-Smorodinsky solution is scale invariant (see Theorem 4.1) we can assume without loss of generality that $d(B)=d(C)=(0,0)$. 
Define $H(C)$ to be the maximal point of $S(C)$ on the line segment with $d(B)$ and $u(S(B), d(B))$ as endpoints. As in the proof of Theorem 3.2, it can be shown that

$$
H(C) \leq K S(B)
$$

From Lemma 2.4 it follows that $H(C)$ is Pareto optimal. Suppose that $K S_{1}(C)>H_{1}(C)$. Then, $K S_{2}(C)=\frac{u_{2}(C)}{u_{1}(C)} K S_{1}(C) \geq \frac{u_{2}(B)}{u_{1}(B)} K S_{1}(C)>\frac{u_{2}(B)}{u_{1}(B)} H_{1}(C)=H_{2}(C)$. This contradicts the Pareto optimality of $H(C)$. It follows that

$$
K S_{1}(C) \leq H_{1}(C)
$$

From (5) and (6),

$$
K S_{1}(C) \leq K S_{1}(B)
$$

which was to be proved.

Proof of Theorem 4.1. Symmetry, scale invariance and individual monotonicity of the KalaiSmorodinsky solution are immediate. (Weak) Pareto optimality follows from Lemma 2.4.

Let $F$ be a bargaining solution which satisfies symmetry, scale invariance, weak Pareto optimality and individual monotonicity. We show that $F=K S$. Our proof deviates from the proof in Kalai and Smorodinsky [5] because also certain nonconvex feasible sets are permitted.

Let $(S, d) \in \mathbb{S}$. Define $T=\{a s+b: s \in S\}$ with $a=\left(\frac{1}{u_{1}(S, d)-d_{1}}, \frac{1}{u_{2}(S, d)-d_{2}}\right)$ and $b=-a d$. Then $(T, 0) \in \mathbb{S}$, (where we write 0 instead of $(0,0))$ and scale invariance implies

$$
\begin{aligned}
K S(T, 0) & =a K S(S, d)+b \text { and } \\
F(T, 0) & =a F(S, d)+b .
\end{aligned}
$$

So it is sufficient to prove that $K S(T, 0)=F(T, 0)$. Note that $u(T, 0)=(1,1)$ and, therefore,

$$
K S_{1}(T, 0)=K S_{2}(T, 0)
$$

Let $C=\left(A, \bar{a}, R D U_{1}, R D U_{2}\right) \in \mathbb{B}$ such that $T=S(C)$ and $0=d(C)$. Let $U_{1}$ and $U_{2}$ be the associated utility functions.

First consider the case that $u(T, 0)=(1,1) \in T$, hence $K S(T, 0)=(1,1)$. Since, by Lemma 2.4, $P(T)=\{(1,1)\}$, there must be an $a \in A$ with $(1,1)=\left(R D U_{1}(a), R D U_{2}(a)\right)$. Define $C^{\prime}=$ $\left(\{\bar{a}, a\}, \bar{a}, R D U_{1}, R D U_{2}\right) \in \mathbb{B}$ where $R D U_{1}$ and $R D U_{2}$ are the restrictions of the rank dependent utility functionals in $C$. With $L:=S\left(C^{\prime}\right)$, we have $F(L, 0)=(1,1)$ by weak Pareto optimality of 
$F$. By individual monotonicity of $F$ applied to $L \subseteq T$ we have $F(T, 0) \geq F(L, 0)=(1,1)$, hence $F(T, 0)=(1,1)=K S(T, 0)$. This completes the proof of the theorem for this case.

Second and last, consider the case that $u(T, 0)=(1,1) \notin T$, hence $K S(T, 0)<(1,1)$. By Lemma 2.4 there is a function $f$, the graph of which coincides with $P(T)$. Define $y:=\left(\min \left\{x_{1}\right.\right.$ : $\left.x \in T\}, \min \left\{x_{2}: x \in T\right\}\right)$. Let $B=\left(\bar{A},(0,0), \overline{R D U}_{1}, \overline{R D U}_{2}\right)$, where

$$
\bar{A}=\left\{\left(U_{1}(a), U_{2}(a)\right): a \in A\right\} \cup\left\{y,\left(y_{1}, \max \left\{x_{2}: x \in T\right\}\right),\left(\max \left\{x_{1}: x \in T\right\}, y_{2}\right)\right\},
$$

with the utility functions $\bar{U}_{i}$ defined by $\bar{U}_{i}(x)=x_{i}$ for every $x \in \bar{A}$, and with weighting functions $\bar{w}_{i}$ equal to those in $C$. Then $d(B)=0, P(B)=P(T)$, and

$$
S(B)=\left\{x \in \mathbb{R}^{2}: y \leq x \leq x^{\prime} \text { for some } x^{\prime} \in P(T)\right\} .
$$

Denote $\widetilde{T}=S(B)$. Then $u(\widetilde{T}, 0)=(1,1)$ and $T \subseteq \widetilde{T}$, so by individual monotonicity of $F, F(\widetilde{T}, 0) \geq$ $F(T, 0)$. Since $K S(\widetilde{T}, 0)=K S(T, 0)$, it is sufficient to show that $F(\widetilde{T}, 0)=K S(\widetilde{T}, 0)$ : then $K S(T, 0) \geq F(T, 0)$ which implies $K S(T, 0)=F(T, 0)$ since $K S(T, 0)<(1,1)$.

Let $z:=K S(\widetilde{T}, 0)\left(\right.$ so $z_{1}=z_{2}$ ) and let $f:[0,1] \rightarrow[0,1]$ be a continuous strictly decreasing function with $(t, f(t)) \in \widetilde{T}, f\left(z_{1}\right)=z_{2}$, and $f(t)=f^{-1}(t)$ for every $t \in[0,1]$. Then $f(0)=1$, $f(1)=0$, and by Lemma 2.5 and its proof there is a $(V, 0) \in \mathbb{S}$ with $P(V)$ equal to the graph of $f$ and $V=\left\{x \in \mathbb{R}^{2}: 0 \leq x \leq x^{\prime}\right.$ for some $\left.x^{\prime} \in P(V)\right\}$. Because $V$ is symmetric by construction, symmetry and weak Pareto optimality of $F$ imply $F(V, 0)=z$. Since $u(V, 0)=(1,1)$ and $V \subseteq \widetilde{T}$, individual monotonicity of $F$ implies $F(\widetilde{T}, 0) \geq F(V, 0)=z$, hence $F(\widetilde{T}, 0)=z=K S(\widetilde{T}, 0)$ since $z \in P(\widetilde{T})$. This completes the proof of the second case, and hence of the theorem.

\section{References}

1. A. Burgos, S. Grant and A. Kajii, Bargaining and Boldness, Games Econ. Behav. forthcoming.

2. A. Chateauneuf and M. Cohen, Risk Seeking with Diminishing Marginal Utility in a NonExpected Utility Model, J. Risk Uncertainty 9 (1994), 77-91.

3. S. Chew, E. Karni and Z. Safra, Risk Aversion in the Theory of Expected Utility with Rank Dependent Preferences, J. Econ. Theory 42 (1987), 370-381.

4. V. Crawford, and H.R. Varian (1979), Distortion of Preferences and the Nash Theory of Bargaining, Econ. Letters 3 (1979), 203-206. 
5. E. Kalai and M. Smorodinsky, Other Solutions to Nash's Bargaining Problem, Econometrica 43 (1975), 513-518.

6. E. Kalai and R.W. Rosenthal, Arbitration of Two-Party Disputes Under Ignorance, Int. J. Game Theory 7 (1978), 65-72.

7. Y. Kannai, Concavifiability and Constructions of Concave Utility Functions, J. Math. Econ. 4 (1977), 1-56.

8. R.E. Kihlstrom, A.E. Roth, and D. Schmeidler, Risk Aversion and Solutions to Nash's Bargaining Problem, in "Game Theory and Mathematical Economics" (O. Moeschlin and D. Pallaschke, Eds.), North Holland, Amsterdam, 1981.

9. J.F. Nash, The Bargaining Problem, Econometrica 18 (1950) 155-162.

10. H. Peters, A Criterion for Comparing Strength of Preference, with an Application to Bargaining, Operations Research 40 (1992), 1018-1022.

11. J. Quiggin, A Theory of Anticipated Utility, J. Econ. Behav. Organ. 3 (1982) 324-344.

12. A.E. Roth and U.G. Rothblum, Risk Aversion and Nash's Solution for Bargaining Games with Risky Outcomes, Econometrica 50 (1982), 639-647.

13. A. Rubinstein, Z. Safra and W. Thomson, On the Interpretation of the Nash Bargaining Solution and its Extension to Non-expected Utility Preferences, Econometrica 60 (1992), $1171-1186$.

14. S. Safra and I. Zilcha, Bargaining Solutions without the Expected Utility Hypothesis, Games Econ. Behav. 5 (1993), 288-306.

15. Z. Safra, L. Zhou and I. Zilcha, Risk Aversion in the Nash Bargaining Problem with Risky Outcomes and Risky Disagreement Points, Econometrica 58 (1990), 961-965.

16. J. Shalev, Loss Aversion and Bargaining, CORE Discussion Paper, 1997.

17. J. Sobel, Distortion of Utilities and the Bargaining Problem, Econometrica 49 (1981), 597619. 
18. J. Sobel, Manipulation of Preferences and Relative Utilitarianism, Games Econ. Behav. 37 (2001), 196-215.

19. A. Tversky and D. Kahneman, Advances in Prospect Theory: Cumulative Representation of Uncertainty, J. Risk Uncertainty 5 (1992), 297-323.

20. M.E. Yaari, Some Remarks on Measures of Risk Aversion and on Their Uses, J. Econ. Theory 1 (1969), 315-329.

21. M.E. Yaari, Univariate and Multivariate Comparisons of Risk Aversion: A New Approach, in "Uncertainty, Information and Communication, Essays in Honor of Kenneth J. Arrow" (P. Heller, R.M. Starr, and D.A. Starrett, Eds.), Vol. III, 173-187, Cambridge University Press, Cambridge, 1986.

22. O. Volij and E. Winter, On Risk Aversion and Bargaining Outcomes, Working Paper, Hebrew University, 2000. 